\title{
Cloud Computing: A Survey on Cloud Computing
}

\author{
Rydhm Beri \\ M.Sc (CS) Student \\ Department of Computer Science \\ BBK DAV College for Women, Amritsar
}

\author{
Veerawali Behal \\ Assistant Professor \\ Department of Computer Science \\ BBK DAV College for Women, Amritsar
}

\begin{abstract}
The internet is marvelous technology of the computing world. The most exhaustive technology that internet dispense, is the Cloud Computing. It provides instrumental values to the customers and cloud service providers. With the advent of cloud computing the client uses the resources like hardware, software or storage, that are offered by other organization, instead of buying their own resources. However, these cloud services are provided by the cloud service providers by means of virtual machines. This study proposed the various aspects related to cloud computing technology. This paper includes the service model that reveals the various cloud services provides to different types of clients according to their requirements. This study provides the details of the layers in the architecture of cloud computing. Moreover, this study also includes the information about the various types of clouds.
\end{abstract}

\section{General Terms}

Cloud Environment, Architecture of Cloud Computing, Service Models

\section{Keywords}

Cloud Computing, Architecture, Service Models, Types of Clouds, Advantages.

\section{INTRODUCTION}

Internet is the most precious technology that all individuals are using today. Internet provides us various technologies that have been used by many people from many years. Some of the technologies include WWW (World Wide Web), Cloud Computing, IRC (Internet Relay Chat). Cloud computing is the most widely used and new style of technology in today's computing world. Cloud computing is a model for enabling ubiquitous, convenient, on-demand network access to a shared pool of configurable computing resources (e.g., networks, servers, storage, applications, and services) that can be rapidly provisioned and released with minimal management effort or service provider interaction [5][6][7].

Cloud computing is the term used to refer to the resources that are provided over the internet. The organization provides the resources on rent basis which costs less. Cloud Computing is new style of computing which is getting progress constantly. Cloud Computing includes computational and storage services as pay you go model [1].

This technology is named as cloud computing, because the term internet or network is often represented as a cloud diagrammatically and cloud computing is the use of internet to provide various computing resources to different people with different requirement at different places.

The main goal of the cloud computing is to distribute the work to different group of individuals, or to work collaboratively with others and use the resources of other organizations to perform large scale computation. Cloud Computing deals in various disciplines like Virtualization, Load Balancing, interoperability, quality of services.

Suppose you are a network administrator and you have the responsibility to fulfill the needs of the employees such as Computer, Software, or hardware that they require to perform their work. If you buy the resources, you have to take care about various matters, such as infrastructure, or hardware require to be setup, or software to be installed on individual computer or the storage space according to the requirement and certification details of the various resources. This will leads to increase in cost and strenuousness in maintenance. The better solution to reduce the cost is that, instead to buy the resources or infrastructure you can use the cloud services this will lead to reduce the cost and decrease the maintainability. By using cloud you do not need to install the software on individual computers or not require buying the storage space for each computer. You can simply use the services of cloud with the help of internet. This paper is organized as follows:

- Section II- Review of Related Work

- $\quad$ Section III- Service Models

- $\quad$ Section IV-Cloud Computing Architecture

- Section V-Types of Cloud

- $\quad$ Section VI- Advantages

- $\quad$ Section VII- Disadvantages

\section{REVIEW OF RELATED WORK}

Several studies have been reported that have been focused on the cloud environment. In this section, various papers are studied that are related to cloud computing and various issues regarding clouds.

David C. Wylid describes that cloud computing encompasses wide range of services that are hosted in a variety of ways, according to the nature of services the security needs to be contrasted [9]. Ahmed E. Youseff and Manal Alageel describes cloud computing as a model for enabling convenient, on demand network access to a shared pool of configuration computing resources that can be released with minimal effort or service provider interaction[10]

\section{SERVICE MODEL}

Cloud computing service refers to the various services provided by the cloud service providers. The services of the cloud computing can be divided in the three ways. 


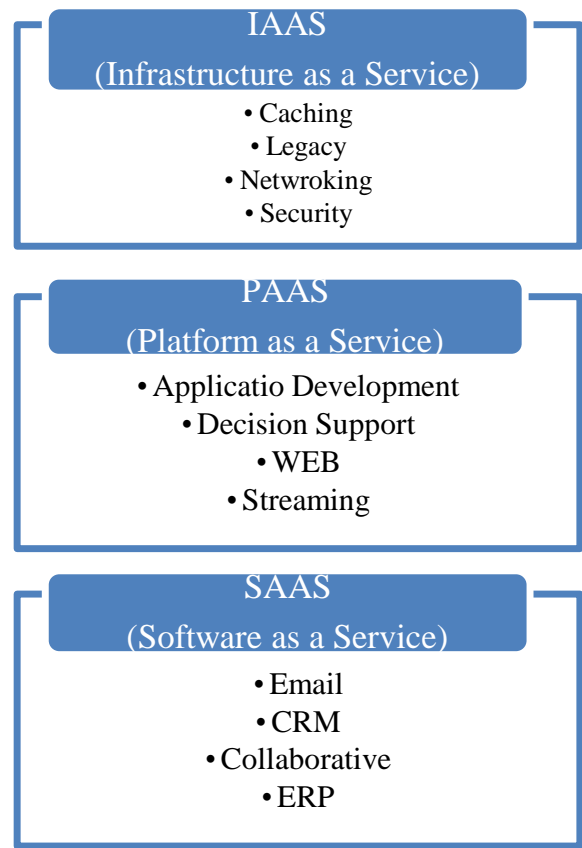

Figure: 1 Service Models of Cloud

\subsection{IaaS(Infrastructure as a Service)}

Iaas refers to the services of infrastructure provided by the cloud service providers. The cloud service provider in this case, provides the Virtual servers, Load Balancers, or storage to the clients and client need to install their own software or operation system on those severs. Here, the responsibility to manage the server is of the cloud service providers, instead of client; however, the responsibility to manage the software or their certification is fully dependent on the client. In other words, the facility provided to the customer is to lease processing, storage, and other fundamental computing resources [8].

\subsection{Paas (Platform as a Service)}

PaaS refers to the development services provided by the cloud service providers to the client. The cloud service providers in this case provides the client a Development environment such as Database, Storage space, by which client can fulfill their development needs. In this case client does not need to install the database software or other software required in development process; instead they can use the development resources of cloud service providers over the internet. The development resources may consume more storage space as compared to other resources. By using these resources of cloud one can avoid the cost of buying the large storage space required to install these application. PaaS refers to providing Platform layer resources, including operating system support and software development framework [3]. Key Examples are GAE, Microsoft Azure [4].

\subsection{SaaS (Software as a Service)}

SaaS refers to the software services provided by the cloud service providers to the number of clients. The cloud service provider in this case provides software such as Email facility to client. In this case, the client does not need to maintain or install the software on their own hardware machines, instead they simply need to pay some amount in the form of rent to the services providers and use their services. SaaS is a model of software deployment where an application is hosted as a service provided to customers across the Internet [2].

\section{CLOUD COMPUTING ARCHITECTURE}

The architecture of the cloud computing is served as the layered model. In general the cloud computing architecture layers are divided into four parts: hardware layer, Infrastructure layer, platform layer, application or software layer

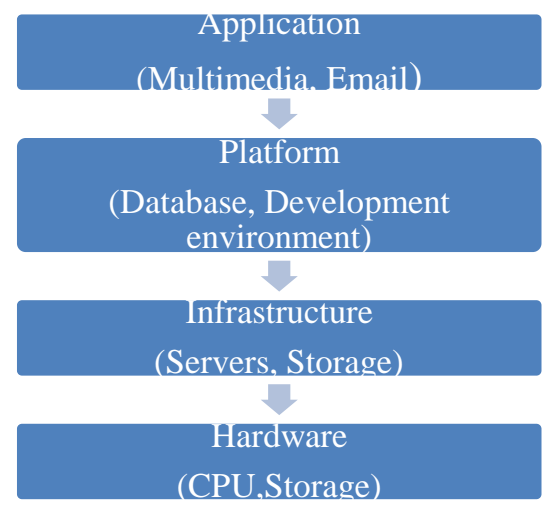

Figure 2: Layered Model of Cloud Computing

\subsection{Layer of Hardware}

The responsibility of this layer is to manage the various hardware devices required in cloud computing. The various devices include storage, processors, routers, switches etc. In practice, the hardware layer is implemented in data centers. A data center usually contains thousands of servers that are organized in racks and interconnected through switches, routers or other fabrics [3].

\subsection{Layer of Infrastructure}

The responsibility of infrastructure layer is to manage the virtual servers, storage media and balance the different nodes to effectively resource utilization or to enhance the response time of the different nodes; this can be done by load balancers.

\subsection{Layer of Platform}

The responsibility of the platform layer is to manage the development resources such as databases, operating systems or other development applications like java, oracle, etc. It helps to reduce the burden of the client to manage heavy storage space required to maintain the software.

\subsection{Layer of Software}

The responsibility of the software layer is to provide the various software services to the clients such as Email services or document applications. This will leads to reduce the burden of the client to buy the software and upgrade their license and install on to the individual machines or manage that software at a single server. This layer helps to provide services like Email services, accounting software or the editing software.

\section{TYPES OF CLOUD}

The cloud can be classified into three categories

. Table 1: Types of Cloud and Issues related to clouds

\begin{tabular}{|c|c|c|}
\hline Cloud & Cost Issue & Security \\
\hline Public Cloud & Setup: & Low \\
& Highest & Security \\
& Usage: Low & \\
\hline Private Cloud & Setup: High & High \\
\hline
\end{tabular}




\subsection{Public Cloud}

It is a cloud in which the cloud service provides services of the various resources to the general public. This reduces the client's burden to invest initial capital investment to buy infrastructure and management of the resources required to establish an infrastructure. The security involved in using the public cloud is lesser as compared to other clouds as anyone in the general public can have access to the services of cloud. There is always a threat to security present in the public cloud.

\subsection{Private Cloud}

Private Clouds referred to the clouds that are organized and managed by the organizations and provides the cloud services to the client located at different locations. These clouds are generally referred to as the internal clouds. Private clouds offer high reliability and security as compared to the other types of clouds.

\subsection{Hybrid Clouds}

These types of cloud combine the capabilities of both the public and private clouds and try to remove the deficiencies of those clouds. In a hybrid cloud the part of the service infrastructure runs in private clouds while remaining cloud runs in public clouds.

\section{ADVANTAGES}

The cloud computing offer the several advantages over the other services of the networks. This section includes some of the advantages of cloud computing.

\subsection{Reduced Cost}

Cloud computing reduces cost factor required for the client by reducing the initial capital investment and management of the resources. As the Cloud Service providers needs to owns and maintain all the computing resources like software, storage and their related issues

\subsection{Better Performance}

In cloud computing the application are typically runs on the cloud servers. Users need not to install heavy software on their own computers of less processing speed. This will lead to increase the performance of the computer at the client side

\subsection{Reduced IT Infrastructure Cost}

Cloud services provides infrastructure as a services to the users, this will leads to reduce the installation cost for the infrastructure by paid by the client at the time of physical server establishment.

\subsection{Unlimited Storage Capacity}

With the help of cloud services the client can use the unlimited storage capacity provided by the cloud service provide. When the storage capacity of the client increases, he/she will simply pay a little more to use the large storage of the cloud server because the installation of large storage costs more as compared to lease the storage.

\subsection{Universally Availability of the Data}

By using the cloud computing services one can access their data anywhere and when required. Client simply needs to login to their cloud account where they store their data.

\subsection{Lesser Training is Required}

It takes fewer learning curves for the individual to learn about the hardware and software issues.

\subsection{Availability of Latest Software}

With the help of cloud services one can use the latest software over the internet. The client does not required to maintain the software on their machines and they do not required buying certification for the software.

\subsection{Increased Group Collaboration}

Cloud services increases the group collaboration. The various individual at different location can work collaboratively at same project or topic. Sharing of the documents with different groups becomes easy with the help of cloud services.

\section{DISADVANTAGES}

Despite the various advantages of the cloud computing there are several disadvantages of the cloud computing. Some of the disadvantages include:

\subsection{Requirement of Internet Connection Constantly}

To use the various services of cloud one continuously requires an internet connection. When the internet connection is down no one can use the services of the cloud offline.

\subsection{Does not work well with slow Internet Connection}

The slow internet services such as dial-up connection makes it difficult or impossible to use the cloud services. This may cause very time consuming process to use the documents stored at cloud servers.

\subsection{Lesser Security}

The use of Public cloud often leads to lesser security as it can be accessed by the general public and there may some hackers involved in general public that can cause damage to data or misuse the data.

\section{CONCLUSION}

In nutshell, the study tries to explain that, Cloud computing is the latest technology of internet. This study explains the various service models of cloud computing, by which evaluation is made different categories of services provided in this technology. This study summarized the layered architecture of the cloud and evaluates how the cloud services are accessed. Clouds are categorized as private cloud, public cloud, and hybrid cloud. This categorization is done according to the organization and security needed by different individuals.

\section{REFERENCES}

[1] Veerawali Behal, Anil Kumar: "Cloud Computing: Performance Analysis of Load Balancing Algorithms in Cloud heterogeneous Environment, IEEE, Sept. 2014.

[2] E Kusuma Kumari et al."Cloud Computing: An Overview", Journal of Theoretical and Applied Information Technology.

[3] Qi Zhang et al."Cloud Computing: state-of-the-art and research challenges", The Brazilian Computer Society 2010, Apr 2010.

[4] Bharskar Prasad Rimal, Eunmi Choi, "A taxonomy and Survey of cloud computing system",2009 Fifth International Joint Conference on INC, IMS and IDC, published by IEEE Computer Society. 
[5] Peter Mell, Thimothy Grance,"The NIST definition of Cloud Computing", National Institute of Standards and Technology, Special Publication 800-145.

[6] Ahmed E. Youssef et al. "A Framework for Secure Cloud Computing", IJCSI, July 2012.

[7] Prabha Sharma,"Gird Computing Vs. Cloud Computing", International Journal of Information and Computation Technology, ISSN 0974-2239 Volume 3, Number 6 (2013), pp. 577-582.
[8] Ayesha Malik, Muhammad Mohsin Nazir, "Security Framework for Cloud Computing Environment: A Review", Journal of Emerging Trends in computing and information Sciences, March 2012.

[9] David C. Wylid, "The Cloudy Future of Government IT: Cloud Computing and the Public Sector around the World", International Journal of Web and Technology \& Semantic technology, Jan 2010.

[10] Ahmed Youseff and Manal Alageel, "A Framework for Secure Cloud Computing”, IJCSI, July 2012. 